\title{
Partitioning Behavior and Interfacial Activity of Phenolic Acid Derivatives and their Impact on $\beta$-Lactoglobulin at the Oil-Water Interface
}

\author{
Alina Bock ${ }^{1,2}$ (D) Ulrike Steinhäuser ${ }^{2} \cdot$ Stephan Drusch ${ }^{1}$ \\ Received: 2 July 2020 / Accepted: 11 December 2020 / Published online: 4 January 2021 \\ (C) The Author(s) 2020
}

\begin{abstract}
Proteins are able to stabilize dispersed food systems due to their amphiphilic nature, acting as emulsifiers. Their interfacial properties can be influenced by different methods, including the formation of protein-phenol nanocomplexes. In this study, the interfacial behavior of phenolic compounds and protein-phenol nanocomplexes was first characterized according to the oil-water partitioning behavior of phenolic acid derivatives according to their molecular structure and its impact on interfacial tension. The influence of the phenolic compounds on protein film formation and its properties by dilatational rheology was then evaluated. The most phenolic acid derivatives are predominantly present in the aqueous phase. Despite their hydrophobic benzene body, weak interfacial activity was observed depending on their chemical structure. This result supports possible protein-phenol nanocomplex formation in the aqueous phase and possible interactions at the oil-water interface. Protein-phenol nanocomplexes showed decreased interfacial adsorption properties and decreased viscoelastic interfacial behavior, depending on the expansion of the delocalized $\pi$-electrons in the phenol.
\end{abstract}

Keywords Phenolic acid derivatives $\cdot \beta$-Lactoglobulin $\cdot$ Partitioning $\cdot$ Interfacial tension $\cdot$ Dilatational rheology

\section{Introduction}

The stability of a dispersed system depends heavily on phenomena occurring at its interface. In terms of system composition, the chemical nature, activity, and film formation of the

Highlights $•$ Phenolic acid derivatives could be interfacial-active

- The oil-water partitioning behavior of phenolic acid derivatives did not correlate with the calculated $\log P$ value

- The number of delocalized $\pi$-electrons in phenolic acid derivatives can affect the interfacial behavior of $\beta$-lactoglobulin

Alina Bock

a.bock@tu-berlin.de

Ulrike Steinhäuser

steinhaeuser@beuth-hochschule.de

Stephan Drusch

stephan.drusch@tu-berlin.de

1 Technische Universität Berlin, Food Technology and Food Material Science, Straße des 17. Juni 135, 10623 Berlin, Germany

2 Beuth Hochschule für Technik Berlin, Food Technology and Food Analysis, Luxemburger Straße 10, 13353 Berlin, Germany emulsifying constituent - as well as the oil-water partitioning and interfacial activity of other constituents - are of major importance. Emulsifiers are either naturally present in the system or added to it in order to facilitate emulsification and increase kinetic stability. In food systems, proteins, and/or low molecular weight emulsifiers are used to stabilize emulsions. The behavior of proteins at the oil-water interface has frequently been investigated [1-5]. Briefly, proteins migrate through the aqueous phase to the interface, where unfolding takes place. Patches with predominating hydrophobic properties are exposed to the hydrophobic oil phase, while sequences with a predominance of hydrophilic amino acids remain in the aqueous phase. Rearrangement of the proteins and intermolecular interactions may result in a viscoelastic interfacial film that resists mechanical forces and provides electrostatic and steric stabilization $[4,6]$. However, this process may be influenced by other ingredients in the dispersed system. A wide range of other food constituents shows interfacial activity and thus may be present at the interface together with the protein. These constituents may also interact with proteins when they are in the same (oil or water) phase and change their interfacial behavior. In recent years, research has focused on tailoring more complex structures at the oil-water interface to 
customize functionality, for example to achieve protection and targeted delivery of bioactive ingredients [7-9]. This so-called "interfacial engineering" first aimed at improving the oxidative stability of nutritional oils through the incorporation of hydrolyzed proteins and phenolics in the interfacial film [10-12]. Other approaches dealt with mechanical stabilization of protein films by means of enzymatical and thermal treatment and chemical crosslinking with e.g. aldehydes [13-15]. Tailored protein structures with specific functional properties have been successfully created, such as fibrils and proteinphenol nanocomplexes [16-18].

Fundamental knowledge of the molecular interactions is required for targeted complex formation and functionalization of the oil-water interface. Protein-phenol interactions in aqueous systems are well characterized. Different studies have shown that at low $\mathrm{pH}$ values, the phenolic hydroxyl groups are protonated and non-covalent hydrophobic interactions with proteins may occur and be stabilized by hydrogen bonds [19-23]. In contrast, at alkaline conditions, hydroxyl groups tend to deprotonate, followed by autoxidation of the phenolic compound and quinone formation. Covalent protein-phenol nanocomplexes resulting from the reaction between quinones and proteins amino acid side chains have already been studied [24-26]. The reactivity of the phenolic constituents is affected by various factors including their chemical structure, the partitioning between oil and water phase in the case of a multi-phase system, and $\mathrm{pH}$-value. Furthermore, in previous studies, position and number of hydroxyl groups were identified as relevant structural characteristics for phenol reactivity with proteins [26-28]. In the present study, $\beta$-lactoglobulin was used as a model protein since its interfacial behavior is well characterized. Previous studies on $\beta$-lactoglobulin and phenolic compound (curcumin) (reference) showed that hydrogen bonds, $\pi$-stacking, and other $\pi$-interactions like $\pi$-alkyl, $\pi$-anion, and $\pi$-sigma - as a kind of hydrophobic interactions - are the main forces for this proteinphenol bonding [29].

These in different ways non-covalently interacting protein and phenolic molecules are sometimes called protein-phenol nanocomplexes. This nanocomplexation can be harnessed to create highly precise interfacial properties of the protein in order to stabilize interfaces. Previous studies mainly used tannic acid (a large and complex molecule) and different polyphenols extracted from green tea (catechine-based polyphenol mixtures) to create protein nanocomplexes for interfacial studies. Increased adsorption rate, decreased interfacial pressure, and increased elasticity of the interfacial films were observed for tannic acid-protein nanocomplexes [30]. Green tea polyphenol-protein nanocomplexes showed different interfacial behavior. Interfacial pressure and viscoelastic properties of the interfacial film decreased in comparison with the pure protein, resulting in improved physical stability of oil-water emulsions and oil phase oxidative stability $[17,31]$. The polyphenols become stacked to protein hydrophobic side chains, so the protein hydrophobic domains decrease their hydrophobicity and could not completely rearrange at the interface, which would explain the changed interfacial behavior $[17,32]$. In this context, most studies focused on complex polyphenols and polyphenol mixtures with three hydroxyl groups per phenolic ring, such as tannic acid and epigallocatechin gallate (EGCG) from green tea.

A systematic investigation on the potential of phenolprotein interactions in dispersed systems taking into account the chemical nature of the phenolic constituents is missing in the literature. Concerning the phenolic constituents, the number of delocalized $\pi$-electrons should be varied to investigate the importance of hydrophobic interactions such as $\pi$-bonds in protein-phenol nanocomplexation. Another important factor is the presence of polar substituents as sugars within the chemical structure. Combined with the more hydrophobic phenolic ring, an amphiphilic molecular structure of the phenolic compound may result. Regarding the interfacial stabilization, factors including the partitioning of the constituents within the dispersed system, the impact of molecular interactions on the adsorption onto the interface, and the changes in interfacial film properties over time as reflected by dilatational rheology should be taken into consideration for a systematic approach $[33,34]$.

The present study aimed to investigate the impact of structural characteristics of phenolic acid derivates and the interaction with B-lactoglobulin on partitioning behavior and their interfacial properties in an oil-in-water emulsion. It was hypothesized that:

a) Phenolic acid derivatives with more polar substituents (e.g. sugars) have a higher amphiphilic character. This results in higher interfacial pressure and a greater proportion of the phenolic compound in the aqueous phase than in the hydrophobic phase.

b) Phenolic acid derivatives with larger $\pi$-electron systems interact more with proteins. Phenolic acid derivatives with two or more of such systems enable proteinphenol-protein interactions (non-covalent crosslinking). This results in an increased diffusion time to the interface and a reduced elastic modulus of the interfacial film.

c) Depending on sugar and delocalized- $\pi$ electrons, phenolprotein nanocomplexes may reduce $\mathrm{E}^{*}$ due to hindered protein film formation / protein-protein interactions.

Different phenolic acid derivatives (PADs) - varying in their number of hydroxylic groups and delocalized $\pi$-electrons, their substituents, and their polarity and molecular size - were evaluated. In the first step, the partitioning behavior of phenolic acid derivatives was analyzed in terms of their molecular structure and their impact on interfacial tension. In a second step, the influence of phenolic compounds on protein film formation at the oil-water interface and its properties were studied by dilatational rheology. 


\section{Materials and Methods}

As a model system, purified MCT oil and distilled water were used to create a biphasic system. B-lactogloblin (B-Lg) was used as a model substance since its behavior at the oil-water interface is well characterized $[5,35,36]$. It was isolated from whey protein isolate (Davisco Foods International Inc.; Le Sueur, Minnesota, USA) and purified to $99 \%$ native form as described by Keppler et al. [37]. Eight different phenolic acid derivatives with variance in hydrophilic residues and number of delocalized $\pi$-electrons were used in the present study (Fig. 1).

As their base structure, all chosen phenolic acid derivates have cinnamic acid (1) containing a benzene unit with delocalized $\pi$ electrons, a conjugated double bond in the aliphatic residue, and a carboxylic group that could be esterified. Cinnamic (1), pcoumaric (2), and caffeic (3) acids differ in the number of hydroxyl groups they contain. All of the subsequent structures (38) have two hydroxyl groups in the ortho position in the same manner as caffeic acid but different substituents. Chlorogenic acid (4) has the polar quinic acid as a substituent. Rosmarinic acid (5) and verbascoside (8) contain one caffeic acid residue and another dihydroxy-phenol as a substituent, respectively. The delocalized $\pi$-electrons of the dihydroxy-phenol unit are isolated to other $\pi$-electrons. Chicoric acid (6) and cynarine (7) contain two caffeic acid residues, in which the delocalized $\pi$-electrons are conjugated with $\pi$-electrons of the aliphatic residues. Cynarine (7) and verbascoside (8) are especially esterified with polar substituents such as quinic acid and rhamnose/glucose. All phenolic acid derivates had a purity $>98 \%$. Caffeic acid, chicoric acid, cynarine, and verbascoside were purchased from $\mathrm{Cfm}$ Oskar Tropitzsch GmbH (Marktredwitz, Germany), p-coumaric acid and rosmarinic acid were purchased from Carl Roth $\mathrm{GmbH}$ \& Co. KG (Karlsruhe, Germany). Chlorogenic acid was purchased from Fisher Scientific GmbH (Schwerte, Germany) and (E)-cinnamic acid from Thermo Fisher GmbH (Kandel, Germany).

\section{$\beta$-Lactoglobulin Solutions}

For the protein solution, $\beta-\lg$ was dissolved in distilled water, stirred at room temperature for $60 \mathrm{~min}$, and stored at $8{ }^{\circ} \mathrm{C}$ overnight. The $\mathrm{pH}$ value was set to 6.0 at room temperature with $0.1 \mathrm{M} \mathrm{HCl}$ and $0.1 \mathrm{M} \mathrm{NaOH}$ (Carl Roth $\mathrm{GmbH},>99.9 \%$, analytical grade, Karlsruhe, Germany).

\section{Phenolic Acid Derivatives Solutions}

For the individual phenol solutions, the phenolic acid derivative (up to $0.2 \mathrm{wt} \%$ ) was dissolved in distilled water using an ultrasonic bath with a controlled water temperature of $40{ }^{\circ} \mathrm{C}$ for $15 \mathrm{~min}$ and stored at $8{ }^{\circ} \mathrm{C}$. Due to cynarine low water solubility, it was diluted (up to $0.1 \mathrm{wt} \%$ ) using an ultrasonic bath at $60{ }^{\circ} \mathrm{C}$ for $45 \mathrm{~min}$.

\section{MCT Oil Purification}

Medium-chain triacylglycerols (MCT-oil, $>99.9 \%$, Witarix MCT 60/40, IOI Oleo GmbH, Hamburg, Germany) were used in this study. To remove low molecular weight emulsifiers and other minor constituents, MCT oil was purified with Florisil ${ }^{\circledR}$ $(100 \%, \mathrm{MgO} \times 3.6 \mathrm{SiO} 2 \times 1.53 \mathrm{OH}$, Carl Roth $\mathrm{GmbH}$, Karlsruhe, Germany) in a ratio of 3:1 (oil: Florisil®). It was

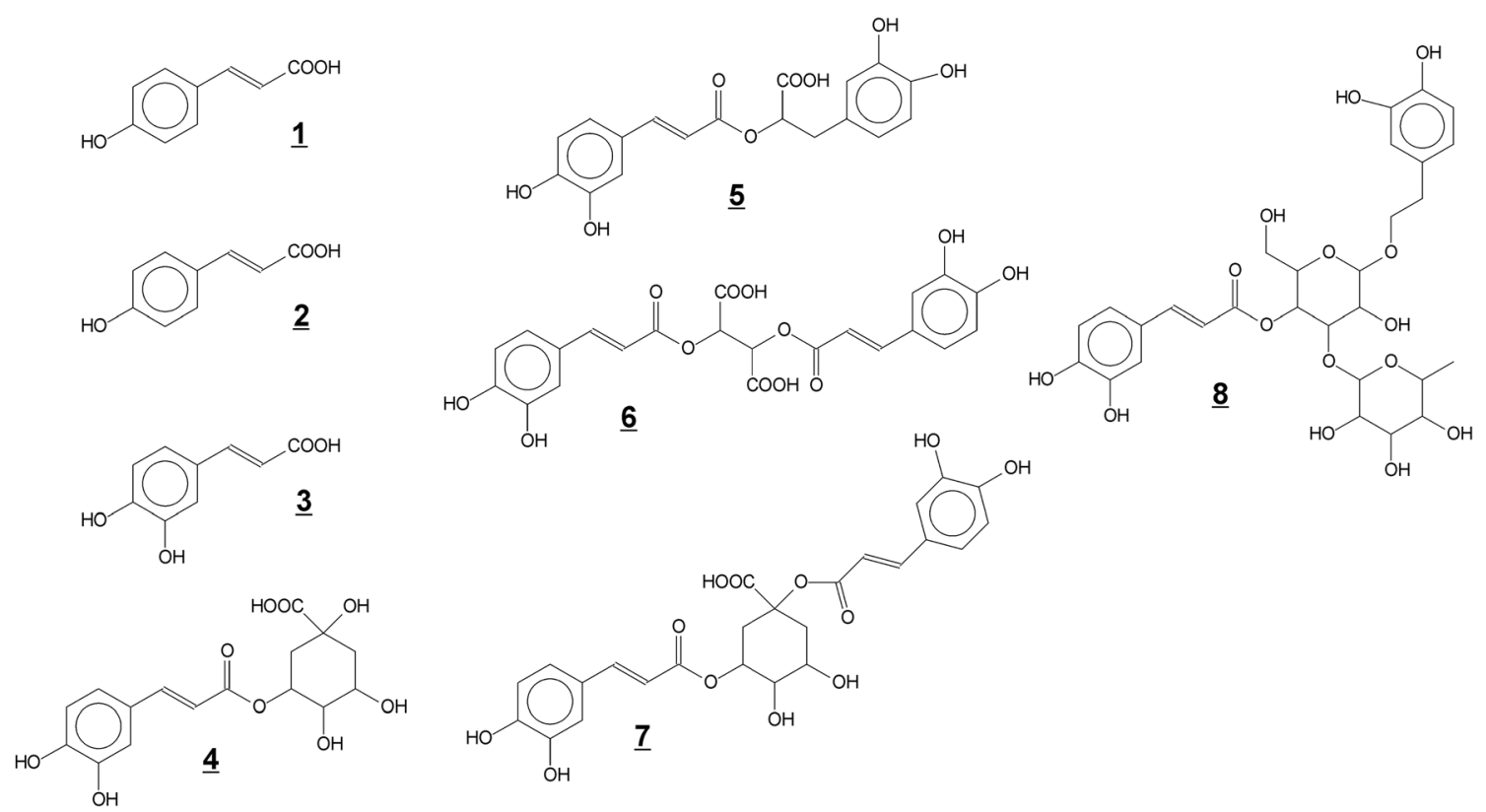

Fig. 1 Phenolic acid derivatives used in the present study: cinnamic acid (1); p-coumaric acid (2); caffeic acid (3); chlorogenic acid (4); rosmarinic acid (5); chicoric acid (6); cynarine (7); verbascoside (8) 
stirred with a magnetic stirrer for a minimum of two hours at room temperature and then centrifuged at 10,000 $\mathrm{g}$ for $45 \mathrm{~min}$. The oil was decanted and its purity was controlled by measuring the interfacial tension against water with a drop tensiometer PAT1-M (Sinterface GmbH, Berlin, Germany). The pure MCT oil showed no decrease in interfacial tension for $60 \mathrm{~min}$.

\section{Partitioning Behavior}

Phenolic acid solution ( $0.05 \mathrm{wt} \%)$ was prepared by dissolving phenolic acid derivatives in distilled water. $1 \mathrm{ml}$ solution was mixed with $1 \mathrm{ml}$ MCT oil in $2 \mathrm{ml}$ Eppendorf tubes. The tube was shaken three times with a vortex for $1 \mathrm{~min}$. After the equilibration time (60 $\mathrm{min})$, the tube was centrifuged at $12,857 \mathrm{~g}$ for $5 \mathrm{~min}$. An aliquot of the lower aqueous phase was removed using a Hamilton syringe with a thin cannula in order to avoid the mixture of the phases. The aqueous phase was added to an HPLC vial for analysis. $0.5 \mathrm{ml}$ of the upper MCT oil phase was removed (with distance to the interface) and transferred to a new $2 \mathrm{ml}$ Eppendorf tube for the liquidliquid microextraction of the phenolic acid. In this tube, $1 \mathrm{ml}$ $\mathrm{MeOH}$ (Carl Roth $\mathrm{GmbH},>99.9 \%$, HPLC gradient grade, Karlsruhe, Germany) $80 \%$ was added and shaken three times with a vortex for $1 \mathrm{~min}$, then equilibrated for $60 \mathrm{~min}$ and centrifuged at $12,857 \mathrm{~g}$ for $5 \mathrm{~min}$. An aliquot of the upper methanolic phase was removed and diluted if necessary (when the peak area was not within the range of the HPLC calibration curve) with ultrapure water for HPLC analysis ([38] with modification).

\section{HPLC Analysis}

All phenolic compounds were analyzed by high-performance liquid chromatography (HPLC). An Agilent 1100 series system with a diode array detector was used. The eluent was isocratic with $40 \% \mathrm{MeOH}$ (Carl Roth $\mathrm{GmbH}$, >98\%, HPLC gradient grade, Karlsruhe, Germany) and $0.1 \%$ formic acid (Carl Roth $\mathrm{GmbH},>99.9 \%$, analytical grade, Karlsruhe, Germany) in completely desalinated water. The separating column was a reversed-phase column (Eurospher 100-5 C18, $250 \times 4.6 \mathrm{~mm}$, Knauer $\mathrm{GmbH}$, Berlin, Germany). The run-time was up to $16 \mathrm{~min}$. The phenolic compounds (from the MCT oil and aqueous phase) were quantified using an external calibration curve of the individual compound. The calibration curves are statistically proved for the range of $0.025 \mathrm{mg} / \mathrm{ml}$ to $0.5 \mathrm{mg} / \mathrm{ml}$. The injection volume was $10 \mu \mathrm{l}$. All analyses were performed in triplicates. The analyzed content of phenolic compounds from both phases was compared with the $\log \mathrm{P}$ value. $\mathrm{P}$ is the partition coefficient between hydrophobic and aqueous phase $(\mathrm{P}=$ [hydrophobic]/[aqueous]). The most common hydrophobic phase is octan-1-ol. A positive $\log \mathrm{P}$ value describes a higher content of the compound in the hydrophobic phase. A negative $\log \mathrm{P}$ value indicates a higher content in the aqueous (hydrophilic) phase of a biphasic system. LogP values can be predicted by software, e.g. from ChemAxon (Budapest, Hungary).

\section{Pendant Drop Analysis}

The analyses of the adsorption at the oil-water interface and the interfacial tension of phenolic acid derivatives and their mixture with $\beta$-lg were performed with the drop tensiometer PAT1-M (Sinterface GmbH, Berlin, Germany). For analyzing the pure samples, the protein and phenolic solutions were diluted if necessary with distilled water to get a final content of $0.1 \mathrm{wt} \%$. To prepare the protein-phenol mixture, the protein and the phenolic solutions were mixed in the ratio $1: 1(\mathrm{v}: \mathrm{v})$, with $0.1 \mathrm{wt} \%$ of each compound (only $0.05 \mathrm{wt} \%$ for cynarine due to its low water solubility). For the measurement, a needle $(\mathrm{d}=1.98 \mathrm{~mm})$ was used to generate aqueous droplets with an area of $50 \mathrm{~mm}^{2}$ in MCT oil. A camera recorded the droplet profile at one frame per second. The instrument calculates the interfacial tension by fitting the Young-Laplace equation to the droplet profile [39]. The lowering of the interfacial tension of aqueous solution without interfacial active compounds $\sigma_{0}$ in relation to interfacial tension of the aqueous solution with surface-active compounds $\sigma(t)$ at a set time is defined as interfacial pressure $\pi(t)$.

$\pi(t)=\sigma_{0}-\sigma(t)$

To characterize the adsorption kinetics, the exponential decrease of interfacial tension was fitted with the following equation $\mathrm{f}(\mathrm{x})$ by CurveExpert Basic 2.1.0 (Hyams Development). The initial slope was calculated to compare the interfacial occupancy of the phenolic acid-derivate protein mixtures by taking the first derivative of the fit $\mathrm{f}^{\prime}(\mathrm{x})=0$.

$f(x)=a+b r^{x}+c x$

Based on this equation, the fit of the adsorption process was depicted as interfacial pressure $\pi(\mathrm{t})$. For the calculation of $\pi(\mathrm{t}), 25.5 \mathrm{mN} / \mathrm{m}$ was determined for $\sigma_{0}$ as the interfacial tension from distilled water against pure MCT oil. Small differences in droplet formation time and differences in diffusion time show up in a slight shift on the $\mathrm{x}$-axis of the graph.

The viscoelastic properties of the protein film and its mixtures with phenolic compounds were determined by dilatational rheology. The experimental setup was the same as above. After $12 \mathrm{~h}$ equilibration time, an amplitude sweep was performed in the range from $0.9 \%$ to $6.6 \%$ deformation amplitude as volume-oscillation of the droplet at a frequency of $0.01 \mathrm{~Hz}$. The interfacial viscoelastic (or dilatational) modulus $\left(\mathrm{E}^{*}\right)$ is calculated by the change in the interfacial area (strain) $\Delta \mathrm{A}$, which results in a change in interfacial tension (stress) $\triangle$ IFT. E* comprises an elastic part E'(storage modulus) and a viscous part E" (loss modulus) [40]. The phase 
angle $(\Phi)$ between the oscillating drop area $\Delta \mathrm{A}$ and its resulting change in interfacial tension indicates the interfacial film viscoelasticity. $\Phi$ is $0^{\circ}$ or $90^{\circ}$ when the systems have ideal elastic or ideal viscous behaviors, respectively. The phase angle is calculated by the ratio of E" and E', resulting in $\tan \Phi$. Lissajous plots and S-factor are used to depict dilatational behavior [41] and to describe the shape of Lissajous plots for expansion and compression, respectively. The Sfactor is calculated from slopes of the secant for the point $\mathrm{A} 0$ for expansion and compression $\left(\mathrm{E}_{\mathrm{T}}{ }_{\mathrm{T}}\right)$ and the secant from zero point to $\Delta$ Amax. (E's) (see fig. 2). For a linear viscoelastic system, the S-factor tends to zero. Non-linear viscoelastic properties are expected when $\mathrm{S} \neq 0$. $\mathrm{S}>0$ indicates strainstiffening and $\mathrm{S}<0$ indicates strain-softening of the interfacial film $[42,43]$.

$S=\frac{E^{\prime}{ }_{S}-E_{T}^{\prime}}{E_{S}^{\prime}}$

\section{Statistics}

The calibration data for HPLC analysis was checked on the normal distribution ( $n=10$ for the lowest and the highest calibration point), linearity, and homoscedasticity. For the extracted samples, the accuracy of the method was determined. To indicate the precision, the standard deviations were calculated from the triplicates (three oil samples with phenolic content were extracted). For the statistical analysis of pendant drop data, the data were tested on a normal distribution. A one-way ANOVA with Tukey HSD post-hoc or Scheffe's $(p<0.05)$ test was performed to identify significant differences between the samples. For multiple pendant-drop analyses, the coefficient of variation (CV) for the method $(n<5)$ was determined to calculate the standard deviation in the case of monoplicates.

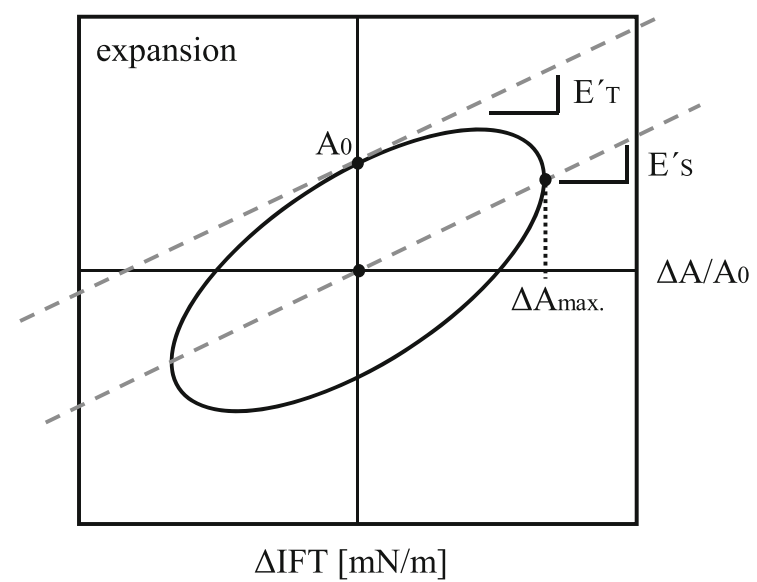

\section{Results \& Discussion}

\section{Oil-Water Partitioning Behavior}

The partitioning of PADs was studied in a biphasic oil-water system (Table 1). The PADs were therefore quantified in the oil and in the water phase by HPLC analysis. The determined partition is used to show differences between oil-water and octanol-water partitioning, which is usually used in literature as " $\log \mathrm{P}$ value" to indicate whether a compound is more present in a hydrophilic or in a hydrophobic phase of a biphasic system.

This analysis showed that more than $60 \%$ of cinnamic acid and coumaric acid, with none or one hydroxyl group per molecule, are present in the oil phase. Hydrophobic interactions predominate between the phenolic compound and the solvent. The addition of a second hydroxyl group already results in a higher partitioning of caffeic acid into the aqueous phase due to an increasing number of hydrogen bonds with water molecules. The presence of polar substituents of the PADs increases the affinity to the aqueous phase. As a consequence, the major part of the compounds (80-100\%) remains in the aqueous phase in the same way as observed for chlorogenic acid, chicoric acid, rosmarinic acid, cynarine, and verbascoside.

The content of cinnamic acid and cynarine could not be fully recovered in the aqueous and oil phase. A possible explanation is the enrichment of these phenolic compounds in the interfacial environment where no phenolic compounds were quantified. Enrichment at the interface could be interesting for the interfacial activity of PADs.

However, in this case the interfacial area was only $0.5 \mathrm{~cm}^{2} /$ $\mathrm{ml}$ of sample, whereas a dispersed system would have a larger interfacial area. In addition, the results of the analysis partly differ from the partition, as expected based on the calculated $\log \mathrm{P}$ values. Negative $\log \mathrm{P}$ values suggest that a compound is mainly present in the hydrophilic phase, while positive $\log \mathrm{P}$ values suggest it is predomininatly present in the hydrophobic

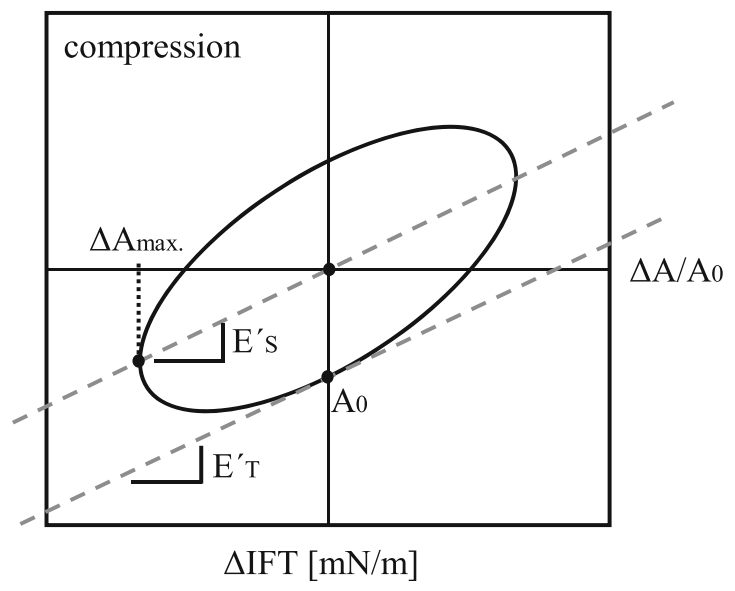

Fig. 2 Calculation of the S-factor (e.g. for an ideal viscoelastic interfacial film, E's = E'T;S = 0), according to [42] 
Table 1 Partitioning behavior of phenolic acid derivatives (0.025 wt $\%)$ in biphasic water-MCT oil systems $(n=3)$.

\begin{tabular}{lllll}
\hline & $\begin{array}{l}\text { Proportion in the } \\
\text { aqueous phase }[\%]( \pm \mathrm{SD})\end{array}$ & $\begin{array}{l}\text { Proportion in the oil } \\
\text { phase }[\%]( \pm \mathrm{SD})\end{array}$ & $\begin{array}{l}\text { Recovery rate }[\%] \text { ( } \pm \text { SD) (for the } \\
\text { phenol extraction out of the oil) }\end{array}$ & $\begin{array}{l}\text { LogP calculated } \\
(\mathrm{ChemAxon})\end{array}$ \\
\hline (E)-cinnamic acid & $18.3( \pm 0.2)$ & $60.8( \pm 13.5)$ & $93.9( \pm 2.5)$ & 2.1 \\
P-coumaric acid & $37.8( \pm 0.6)$ & $63.0( \pm 1.6)$ & $85.9( \pm 1.6)$ & 1.8 \\
Caffeic acid & $65.6( \pm 2.0)$ & $38.3( \pm 3.4)$ & $99.6( \pm 1.6)$ & 1.5 \\
Chlorogenic acid & $102.7( \pm 0.1)$ & $0.2( \pm 0.1)$ & $82.7( \pm 7.5)$ & -0.3 \\
Chicoric acid & $101.3( \pm 0.2)$ & $0.2( \pm 0.1)$ & $102.2( \pm 4.4)$ & 3.0 \\
Rosmarinic acid & $91.1( \pm 1.9)$ & $8.6( \pm 0.2)$ & $93.0( \pm 1.6)$ & 2.0 \\
Cynarine & $81.0( \pm 0.8)$ & $1.4( \pm 0.1)$ & $102.9( \pm 0.4)$ & 0.8 \\
Verbascoside & $99.7( \pm 1.0)$ & $0.2( \pm 0.1)$ &
\end{tabular}

phase. The PADs with only one benzene unit (cinnamic acid, coumaric acid, caffeic acid, or chlorogenic acid) partition as expected based on the $\log \mathrm{P}$ value. In contrast, the higher molecular PADs with two benzene units (chicoric acid, rosmarinic acid, cynarine, or verbascoside) and with positive $\log \mathrm{P}$ values in the oil-water environment displayed a higher affinity to the hydrophilic aqueous phase than to the hydrophobic oil phase (Table 1). A possible explanation is that the phenolic compounds can form hydrogen bonds with the hydroxyl group of the octanol. MCT oil contains fatty acids with a medium chain length from six up to ten carbon atoms and has no hydroxyl groups for interactions, only van der Waals forces are possible. The polarity of a solvent/oil is difficult to investigate because it relates to many factors including imbalances in electronegativity and polarizable functional groups [44]. Therefore it is advisable to characterize the partitioning of a target compound in the respective solvent system rather than using partitioning coefficients from model experiments.

\section{Interfacial Tension of PADs}

The reduction of interfacial tension in the presence of PADs was analyzed as the interfacial pressure after $60 \mathrm{~min}$ of equilibration of the samples (Fig. 3). The PADs with only one benzene unit (the left four columns in Fig. 3) did not show a significant decrease in interfacial tension at the oil-water interface.

The presence of a single caffeic acid residue with a hydrophilic substituent did not generate an interfacial activity, as observed with chlorogenic acid. Compounds with two benzene units (including chicoric acid, rosmarinic acid, cynarine, or verbascoside) display a significant increase in interfacial pressure, depending on additional hydrophilic substituents. The interfacial tension of these compounds decreases with increasing concentration of phenolic acid derivatives (Fig. 4).

This study showed that phenolic compounds can be interfacially active under certain circumstances. However, this is not always the case. Von Staszewski et al. (2014) describes that green tea polyphenols are not interfacial-active [31]. Green tea polyphenols are different phenolic compounds mainly based on the structure of catechine. Catechines contain a benzene unit with two hydroxyl groups in meta-position. This benzene unit has a dihydropyran annelated which has another benzene unit as substituent with two hydroxyl groups in ortho-position. Our study demonstrated that PADs behave very differently than previously described. One hypothesis is that hydroxyl groups, which are distributed over the molecule, may compensate the hydrophobic molecule part (the benzene unit with the $\pi$-electrons). In this case, phenolic compounds are not amphiphilic and not interfacial-active like catechine, caffeic acid, and chlorogenic acid. Moreover, the hydrophobic molecule parts (benzene units with an aliphatic residue) tend to interact with the oil phase and the hydrophilic parts

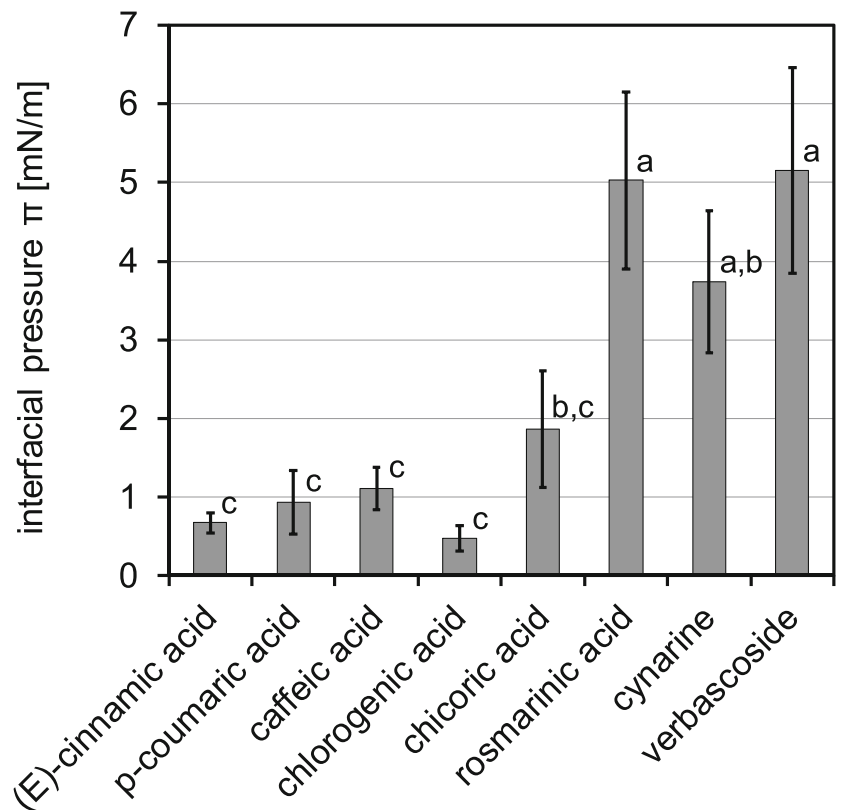

Fig. 3 Interfacial pressure of phenolic acid derivative solutions $(0.05 \mathrm{wt} \%)$ after $60 \mathrm{~min}(n=5)$. The different letters $(\mathrm{a}, \mathrm{b}$, and $\mathrm{c})$ describe the statistical homogeneous groups without significant differences $(p>0.05)$ 


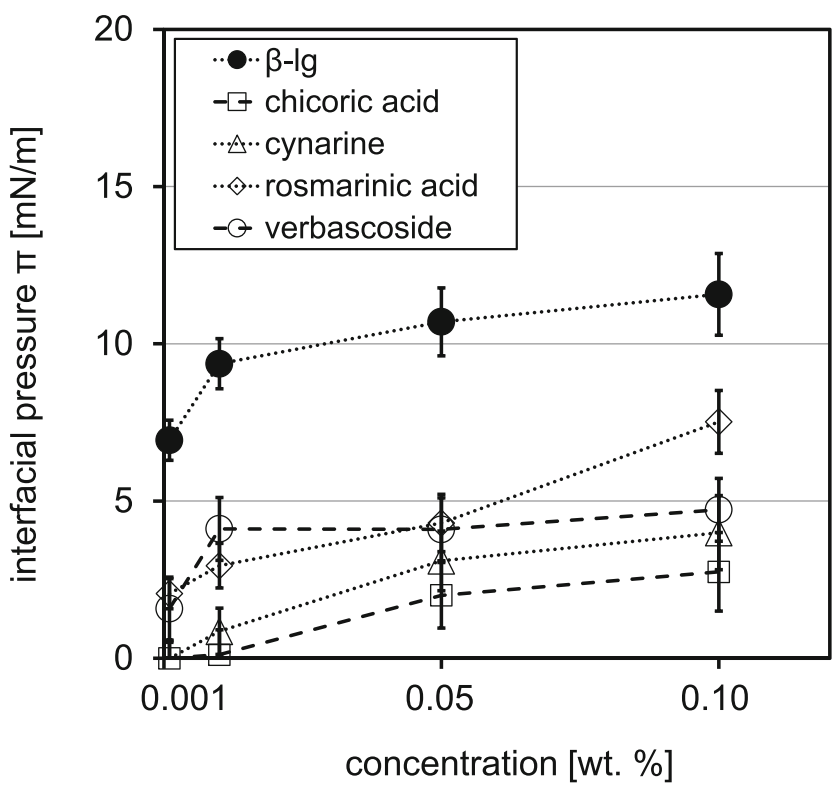

Fig. 4 Interfacial pressure depending on the concentration after $60 \mathrm{~min}$ $(n=2)$ with a calculated standard deviation based on the coefficient of variation $(n=5)$ of each compound; initial interfacial tension was constant at $25.5 \mathrm{mN} / \mathrm{m} \pm 0.3$ for each sample

(substituent) with the aqueous phase. A molecule can have an amphiphilic character if the molecule is large enough to allow spatial separating of the hydrophilic and hydrophilic parts. An increase in interfacial pressure was observed for these molecules. Symmetrical molecular structures hinder the orientation at the interface, as can be observed with chicoric acid. The chemical structure of PADs thus contributes to a significant lowering of interfacial tension (up to $5 \mathrm{mN} / \mathrm{m}$ ), but not comparable with a classical interfacial-active compound such as a tenside or protein (for $\beta-\lg 10 \mathrm{mN} / \mathrm{m}$ ).

\section{Adsorption Processes}

In the previous section, only pure PADs and their behavior were examined. The following section focuses on the impact of PADs on protein properties through protein-PAD nanocomplex formation. First, this nanocomplex formation was studied with isothermal titration calorimetry between caffeic acid (basic structure of PADs) and $\beta$-lg. It showed an entropy-driven interaction between caffeic acid und $\beta$-lg with a molar ratio of up to two caffeic acid molecules to one $\beta$ lactoglobulin molecule. Nanocomplex formation was demonstrated via hydrophobic interactions. A ratio between bound and unbound PADs (caffeic acid with substituents) was not detected, so the interfacial behavior of a mixture with an unknown ratio of $\beta$-lg-PAD nanocomplexes, free $\beta$-lg, and free PADs was studied. The interfacial behavior was studied in terms of the initial slope of the interfacial pressure. The purpose was to characterize the diffusion time of the molecules from the bulk to the interface. Moreover, the focus was on the shape of the curve at the beginning of the adsorption process in order to identify differences in adsorption/unfolding behavior of the $\beta$-lg-PAD mixtures at the interface.

\section{Pendant Drop Tensiometer, Interfacial Tension of PAD-Protein Mixtures}

In general, pure PADs showed a faster diffusion and adsorption at the interface than $\beta$-lg because of their lower molecular size. Moreover, their presence may have an impact on the interfacial behavior of $\beta$-lg.

Accordingly, it was demonstrated that the interfacial tension for protein solution did not show a significant difference when compared with PAD-protein solutions after one hour of analysis (data not shown). However, the maximal adsorption rate (initial slope of interfacial pressure, Fig. 5) and the adsorption and unfolding process (Fig. 6) are affected by the presence of PADs.

For PADs with two caffeic acid residues (conjugated $\pi$ electrons in the benzene unit and its aliphatic rest), a reduced initial slope of the interfacial pressure could be observed (chicoric acid and cynarine) in Fig. 5 with 0.9 and $0.5 \mathrm{mN} / \mathrm{m} * \mathrm{~s}$. For PADs with only one caffeic acid residue (less conjugated $\pi$-electrons), a higher initial slope could be noticed (rosmarinic acid and verbascoside) with $3.8 \mathrm{mN} / \mathrm{m}^{*} \mathrm{~s}$ and pure $\beta-\lg$ with $2.0 \mathrm{mN} / \mathrm{m} * \mathrm{~s}$.

Reduced interfacial pressure in the presence of chicoric acid and cynarine was observed (Fig. 6) when compared to pure $\beta$ - $\lg (\pi=7 \mathrm{mN} / \mathrm{m}$ after $30 \mathrm{~s})$. A small increase in the interfacial pressure was observed for verbascoside and rosmarinic acid (one caffeic acid residue) and protein nanocomplexes ( $\pi=8.5 \mathrm{mN} / \mathrm{m}$ after $30 \mathrm{~s}$ ) and a reduction of

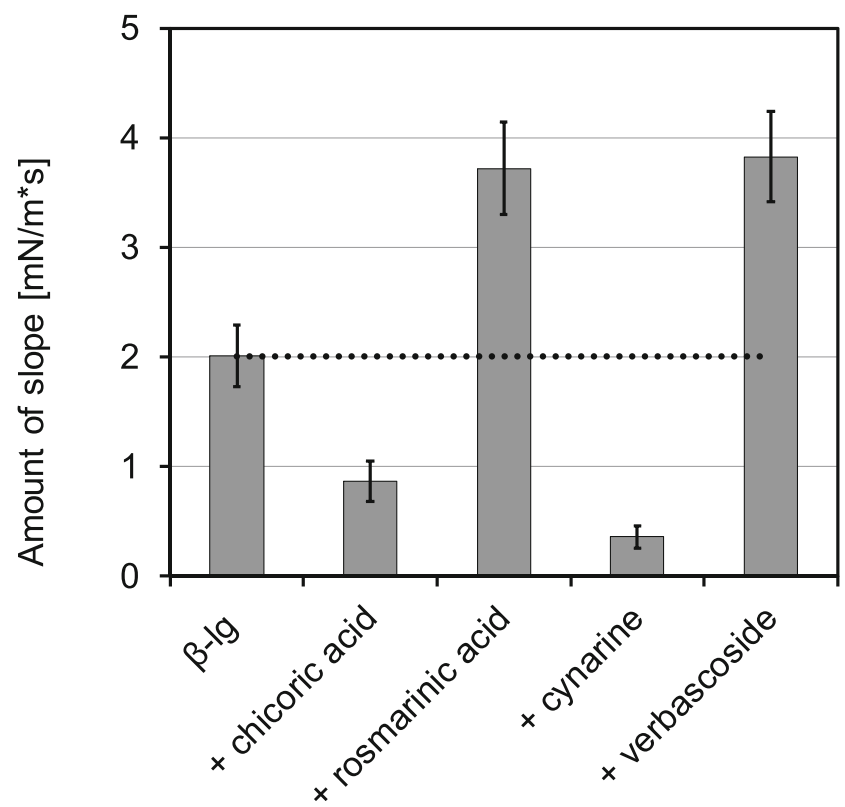

Fig. 5 Initial slope of the interfacial pressure to characterize the adsorption kinetics of $\lg (0.1 \mathrm{wt} \%)$ and its mixtures with phenolic acid derivatives $(0.1 \mathrm{wt} \%),(\mathrm{n}=2)$; the standard deviation was calculated by the coefficient of variation for the method $\mathrm{CV}=11.5 \%(\mathrm{n}=5)$ 
the interfacial pressure was determined for the protein complexes with chicoric acid and cynarine (two caffeic acid residues) ( $\pi=5.5$ resp. $4 \mathrm{mN} / \mathrm{m}$ after $30 \mathrm{~s})$. The initial slope of the fitted curve (Fig. 5) agrees with it.
A higher initial slope of the interfacial pressure for the rosmarinic acid and verbascoside samples could be explained due to unbound PADs with interfacial-active properties. It was observed that rosmarinic acid and verbascoside reduced the
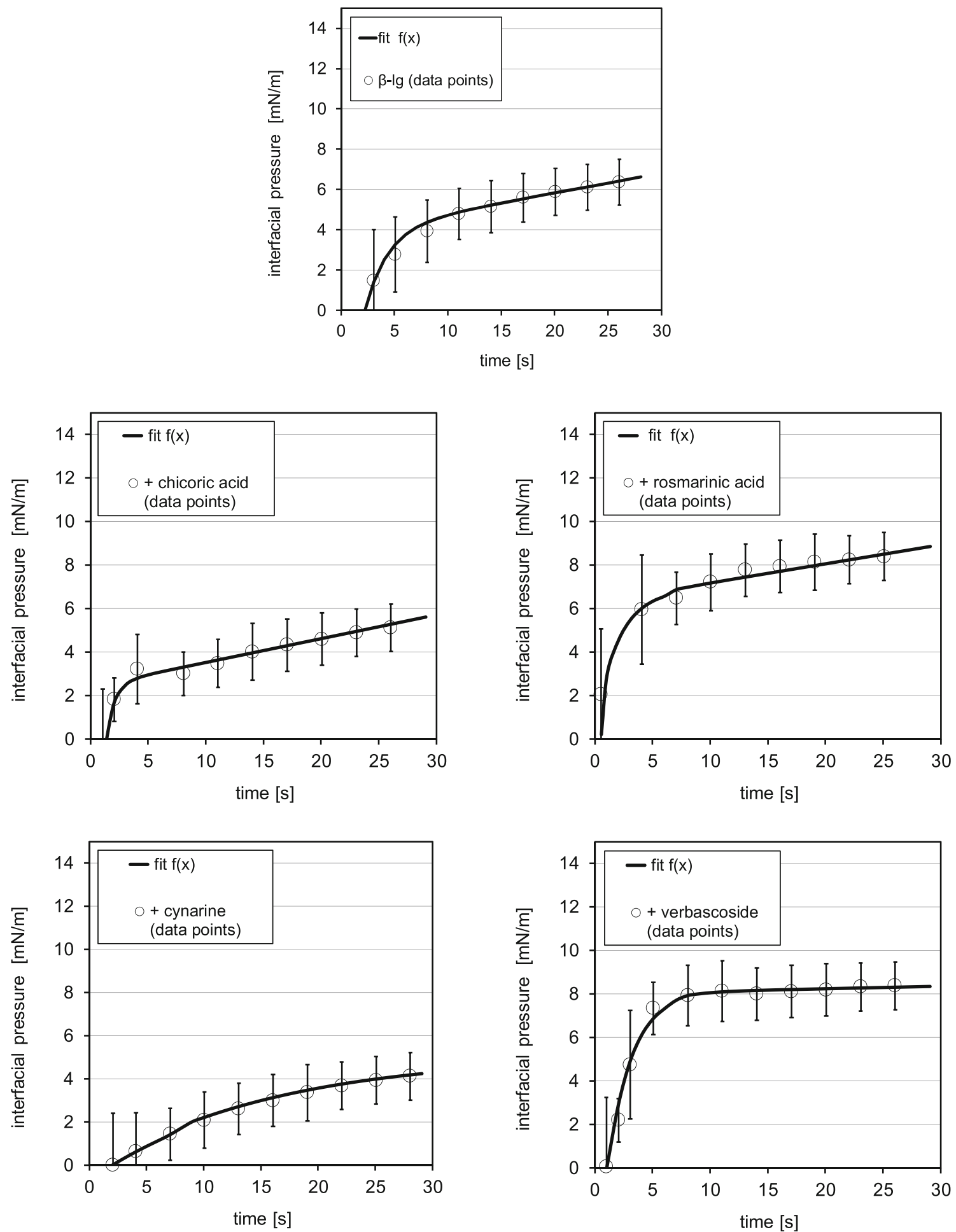

Fig. 6 Fit of the adsorption process over the time of $\beta-\lg (0.1 \mathrm{wt} \%)$ and its mixtures with PADs $(+0.1 \mathrm{wt} \%)$ at $\mathrm{pH} 6.0(\mathrm{n}=2)$ with a calculated standard deviation based on the coefficient of variation $(n=5)$ of $\beta-\mathrm{lg}$ 
surface tension in an oil-water system more effectively than the other PADs (Fig. 2). Unbound rosmarinic acid and verbascoside reach the interfacial area before the $\beta$-lg-PAD nanocomplexes, resulting in an increased slope of the initial interfacial pressure compared to $\beta$-lg. In other words, the $\beta$-lg-PAD nanocomplexes need more time to reach the interface, after which the interfacial tension is predominated by $\beta$-lg. PADs with two caffeic acid residues strive more for interaction with the protein and themselves than with the interface because of their higher number of conjugated $\pi$-electrons. Consequently, there is less unbound cynarine and chicoric acid present, which reaches the interface before the $\beta$-lg-PAD nanocomplexes.

The increased adsorption and unfolding time (Fig. 6) for the $\beta$-lg chicoric acid and cynarine nanocomplexes can be traced back to stronger interactions between the $\beta$-lg and chicoric acid or cynarine than with rosmarinic acid or verbascoside. Possible binding mechanisms of $\beta$-lg with phenolic acids and their esters are based on van der Waals forces, hydrogen bonds, and hydrophobic interactions [45]. Chicoric acid and cynarine have more conjugated $\pi$-electrons than rosmarinic acid and verbascoside. This may result in increased hydrophobic interactions that agree with the isothermal titration calorimetry measurement of the caffeic acid "residue" and $\beta$-lg. One hypothesis to explain the increasing adsorption time is the reduction of surface hydrophobicity in the protein. In this case, hydrophobic residues like the hydrophobic "surface pocket 18 " with the hydrophobic amino acid $\mathrm{Phe}_{136}$ or other hydrophobic amino acids like $\mathrm{Ala}_{34 / 37}$, Try ${ }_{61}$, or $\mathrm{Leu}_{156}$ could be blocked because of their interactions with PADs and the hydrophilic parts of the phenol are on the protein surface. Another possibility is the exposure of previous embedded hydrophilic domains of the protein, due to changes in protein conformation. Hydroxyl groups were introduced to the protein surface, which could be detected by multi-spectroscopy methods [27, 31, 45-47]. With a reduced surface hydrophobicity, the protein is hindered from adsorbing at the interface due to less hydrophobic domains for the oil phase. Also, if the proteinphenol nanocomplex is adsorbed at the interface, there could be competing interactions between protein phenol and protein oil, which could increase the unfolding and film-building time at the interface of the protein.

In this study, it was shown that the PADs with more conjugated free electron pairs have greater increases in adsorption time than the other PADs. So it is possible that hydrophobic (or more accurately $\pi-\pi$ - interactions) are important $\beta$-lg-PAD interactions with an impact on the adsorption kinetics. For $\beta$-lg, it is possible to bind PADs inside its hydrophobic calyx, which is unfolding/exposing at the interface. Possible amino acids in the calyx for $\pi$-interactions are $\mathrm{Phe}_{105}, \mathrm{Asn}_{90}, \mathrm{Ile}_{84}, \mathrm{Ile}_{56}, \mathrm{Val}_{43}, \mathrm{Val}_{92}$, and $\mathrm{Met}_{107}$ residues [29]. Interactions in the calyx may have an important impact on the adsorption and unfolding behavior of $\beta$-lg at the interface as demonstrated in this study. Previous studies showed that binding increases with increasing hydroxyl group numbers, while the number of $\pi$-electrons remains constant $[48,49]$. Consequently, it seems that the expansion of $\pi$-electron systems and the number and position of hydroxyl groups have a significant impact on protein-phenol interactions, which results in changed interfacial behavior of the protein.

\section{Viscoelastic Properties of the Interfacial $\beta$-Lg-PAD Film}

Based on the expansion and compression of an interfacial film (dilatational rheology), it is possible to characterize its properties concerning viscoelastic behavior and film stability. Lissajous plots can be used to present the change in interfacial tension depending on the expansion and compression. The protein-PAD mixtures showed a similar primarily linear plot in the Lissajous plot, which indicates an elastic behavior for all frequencies between $0.9 \%$ and $6.7 \%$ (Fig. 7). For a more viscous or viscoelastic behavior, the plot would be more circular or ellipsoid, respectively.

There are no significant differences between the orientation and shape of the plots and the amplitudes at constant elastic part E'(storage modulus) (data not shown for E'). This confirms that the analyzed amplitudes were within the linear viscoelastic regime [42]. $\beta$-lg seems to dominate the viscoelastic properties of the interfacial film due to the similar shape of the Lissajous plot of $\beta$-lg and $\beta$-lg-PAD mixtures.

The S-factor was used to describe the uniformity of the shape of Lissajous plots for expansion and compression. All samples showed an S-factor near zero, which indicates linear viscoelastic behavior of the interfacial film (fig. 8) without any significant strain-stiffening or strain-softening response. There are no significant differences between the samples.

To evaluate the Lissajous plots, different parameters were calculated. The phase angle $\Phi$ between the oscillating drop area $\Delta \mathrm{A}$ and its resulting change in interfacial tension $\Delta$ IFT tends to zero in an ideal elastic system, because there is no phase shift between $\Delta \mathrm{A}$ and $\Delta \mathrm{IFT}$ during oscillation. The low phase angles of $\beta$-lg and its mixtures with PADs indicate a highly elastic interfacial film in all samples (Fig. 9). The lowest phase angles were shown for pure $\beta$-lg and the mixture with rosmarinic acid at low dilatational stress, increasing with higher dilatational stress. The addition of chicoric acid, cynarine, and verbascoside results in an increased phase shift, especially at low dilatational stress. These PADs may interact with the $\beta-\lg$ on two or more binding sites, and the molecule flexibility is lost. This can be compared to an accordion. When the bellow bars are connected, the accordion cannot be fully opened and its elasticity is reduced in comparison to when bars are open. At higher oscillation amplitudes, the stretching forces are higher, "the connection is ruptured," and the elasticity is similar to the system with only pure $\beta$-lg. For molecules like rosmarinic acid, the connection of two protein molecule parts was weaker because of fewer $\pi$-electrons for hydrophobic interactions in the substituent. Therefore, these molecules bind only to one position on the protein and the behavior is similar to pure $\beta$-lg. 

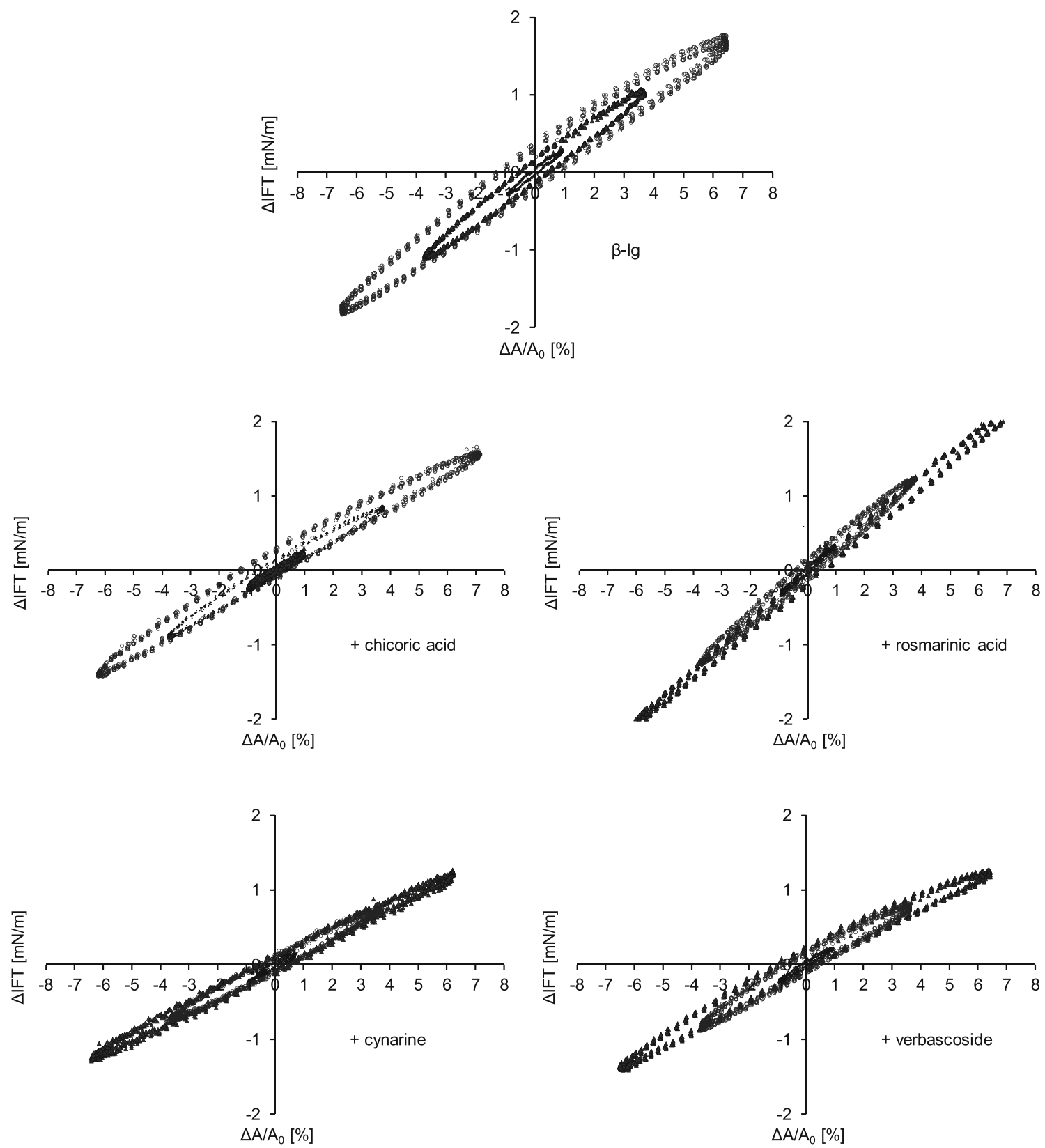

Fig. 7 Overlay of the Lissajous plots with $0.9 \%, 3.5 \%$ and $6.7 \% \Delta \mathrm{A} /$ Ao oscillation

A relevant parameter to describe the interfacial dilatational viscoelasticity is the complex viscoelastic modulus $E^{*}$. This study demonstrated that pure $\beta$-lg has the highest $\mathrm{E}^{*}(\approx 35 \mathrm{mN} /$ $\mathrm{m}$ ) and the lowest $\tan \Upsilon$ (fig. 10). This indicates high interfacial stability with greater elasticity. The $\beta$-lg-PAD mixtures showed a reduction in $\mathrm{E}^{*}$ while increasing $\tan \gamma$ with almost constant $\mathrm{E}^{\prime}$ (data not shown). So the elastic part $\mathrm{E}^{\prime}$ and the stability of these systems decreased due to $\beta$-lg-PAD nanocomplex formation. The presence of two caffeic acid residues (more $\pi$-electrons $\rightarrow$ chicoric acid, cynarine) or an esterified sugar (verbascoside) at the PAD strengthens this tendency (Fig. 10: $\mathrm{E}^{*} \approx 20 \mathrm{mN} / \mathrm{m}$ ). PADs with a large number of delocalized $\pi$-electrons and/or sugar residues limit the elastic properties of $\beta$-lg, which may cause crosslinking reactions or changes in protein folding. In this case, both are based on non-covalent protein-phenol interactions. The impact of delocalized $\pi$-electrons on protein interactions / interfacial film formation is shown by the difference in $\mathrm{E}^{*}$ between rosmarinic acid $\left(\mathrm{E}^{*} \approx 30 \mathrm{mN} / \mathrm{m}\right)$ and chicoric acid $\left(\mathrm{E}^{*} \approx\right.$ $22 \mathrm{mN} / \mathrm{m}$ ). These molecules are similar to differences in the number of $\pi$-electrons. Rosmarinic acid received $18 \pi$ electrons and chicoric acid $24 \pi$-electrons. So the addition of chicoric acid resulted in a more decreased $\mathrm{E}^{*}$ in comparison to rosmarinic acid, which has fewer $\pi$-electrons. Strong hydrophobic protein-phenol interactions such as $\pi$-interactions on two molecule sides (possibility of crosslinking) affect the protein unfolding. Moreover, the rearrangement at the interface and the 


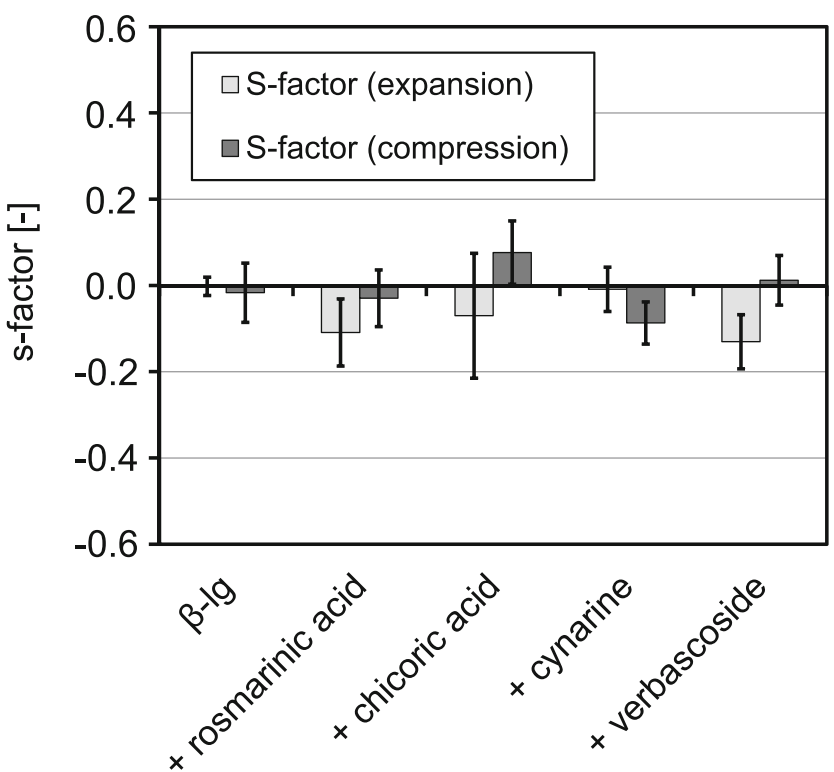

Fig. 8 Complex dilatational moduli of b-lg and its mixtures with phenolic acid derivatives $(0.1 \mathrm{wt} \%+0.1 \mathrm{wt} \%)$, calculated from oscillation cycles $\Delta \mathrm{A} / \mathrm{A} 0=3.6 \%$ and $\mathrm{f}=0.01 \mathrm{~Hz}$

formation of a viscoelastic interfacial film are hindered. The result is a decreasing $\mathrm{E}^{*}$ with less elastic modulus.

The same behavior was observed when the phenolic acid was esterified with a sugar residue and with hydrophilic hydroxyl groups. In number and partition of the $\pi$-electrons, the verbascoside is similar to rosmarinic acid with an additional disaccharide. Thus it is conceivable that the esterified disaccharide residue reduces the elastic properties of the interfacial film due to many factors including (1) sterical hindrances, (2) crosslinking

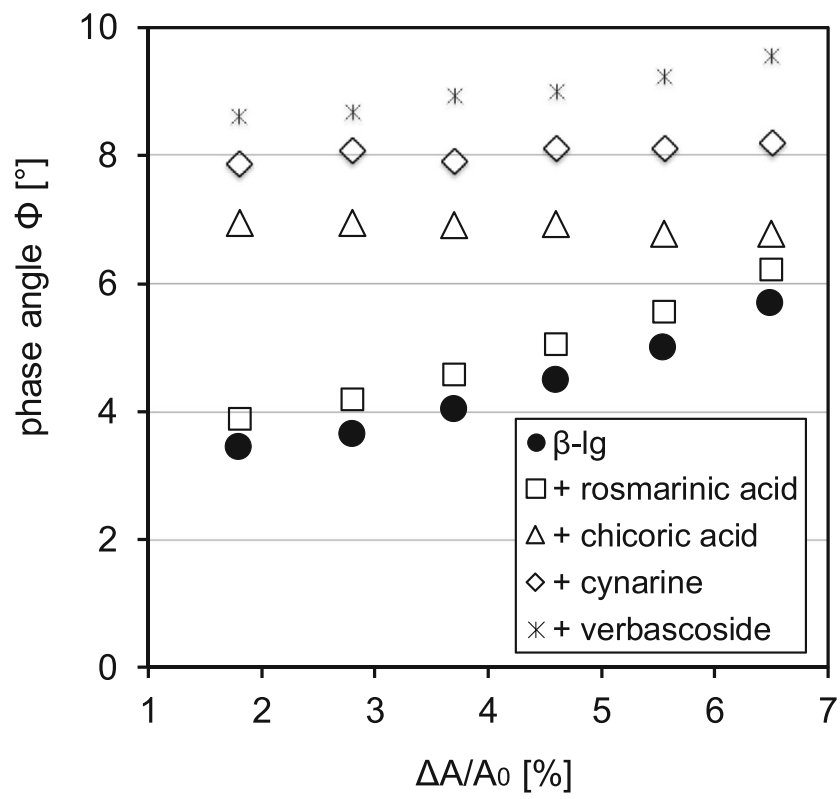

Fig. 9 Phase angle in relation to area change studied by dilatational rheology at a frequency of $0.01 \mathrm{~Hz}$ of $0.1 \mathrm{wt} \% \beta-\mathrm{lg}$ and its mixtures with PADs $(0.1 \mathrm{wt} \%)$

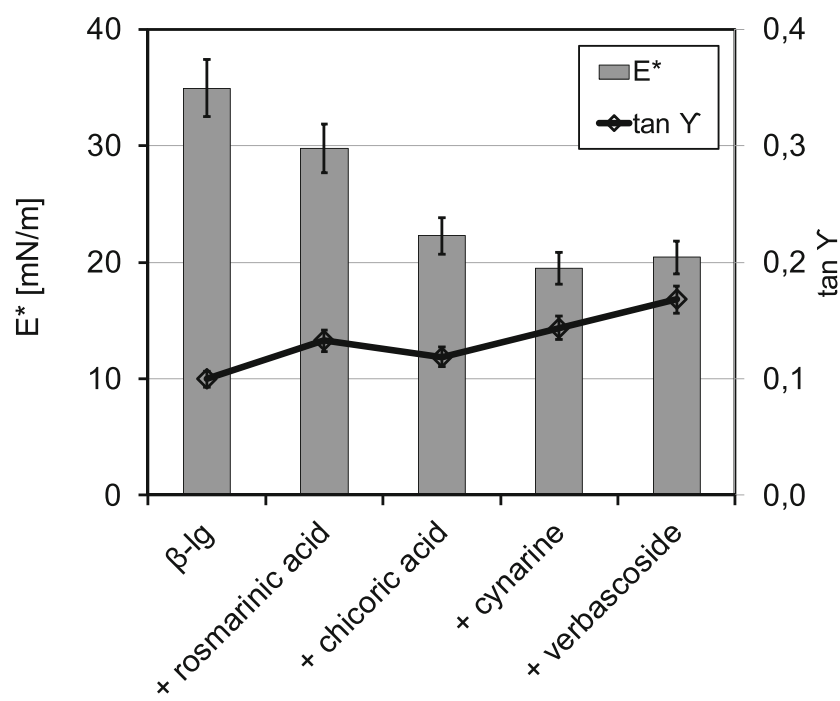

Fig. 10 Primary axis: complex dilatational moduli $\mathrm{E}^{*}$ of b-lg and its mixtures with phenolic acid derivatives $(0.1 \mathrm{wt} \%+0.1 \mathrm{wt} \%)$, calculated from oscillation cycles $\Delta \mathrm{A} / \mathrm{A} 0=6.6 \%$ and $\mathrm{f}=0.01 \mathrm{~Hz}$; secondary axis: $\tan \Upsilon$ as the quotient of $E^{\prime \prime}$ and $E$; the standard deviation was calculated by the coefficient of variation for the method $\mathrm{CV}=7 \%(\mathrm{n}=5)$

via hydrophobic and hydrophilic interactions, (3) hydrogen bonds, and (4) the resulting conformational changes of the protein.

\section{Conclusion}

Phenolic acid derivatives were analyzed for their oil-water partitioning behavior, their potential to reduce the interfacial tension, and their impact on interfacial behavior of $\beta$-lactoglobulin. Phenolic acid derivatives could be weak interfacial-active when they are esterified with a hydrophilic substituent and mainly present in the aqueous phase while having a hydrophobic character. The MCT oil-water partitioning could not be predicted with calculated $\log \mathrm{P}$ values, so it can be concluded that the partitioning varies widely according to the solvents.

For the phenolic acid derivative and $\beta$-lactoglobulin mixtures, it can be assumed that with the increasing number of delocalized- $\pi$-electrons of the phenolic compound, there is an increase of the hydrophobic phenol-protein interactions, resulting in non-covalent crosslinking of the $\beta$-lactoglobulin by phenolic acid derivatives and conformation changes. These changes may lead to an increased interfacial adsorption time, which could be due to reduced surface hydrophobicity and/or a higher hydrodynamic diameter of the $\beta$-lactoglobulin and phenolic acid derivative nanocomplexes. These conformational changes and crosslinking reactions have an impact on the viscoelastic properties of the interfacial films, which have decreasing viscoelastic properties because of reduced elasticity. The presence of sugar residues in the phenolic compounds also affects decreased viscoelastic properties.

Our study built on other studies that analyzed the effect of the number of hydroxyl groups. It further established that the number of delocalized $\pi$-electrons in a phenolic compound 
has a significant impact on the interfacial behavior of $\beta$ lactoglobulin and phenol nanocomplexes.

Supplementary Information The online version contains supplementary material available at https://doi.org/10.1007/s11483-020-09663-7.

Acknowledgments Alina Bock gratefully acknowledges the financial support of the Beuth University of Applied Sciences.

\section{Availability of Data and Material Not applicable.}

Authors' Contributions All authors contributed to the study's conception and design. Material preparation, data collection, and analysis were performed by Alina Bock. The first draft of the manuscript was written by Alina Bock and all authors commented on previous versions of the manuscript. All authors read and approved the final manuscript.

Funding Open Access funding enabled and organized by Projekt DEAL. Alina Bock was financially supported by Beuth University of Applied Sciences.

\section{Compliance with Ethical Standards}

Conflicts of Interest The authors declare that they have no conflict of interest.

Code Availability Not applicable.

Open Access This article is licensed under a Creative Commons Attribution 4.0 International License, which permits use, sharing, adaptation, distribution and reproduction in any medium or format, as long as you give appropriate credit to the original author(s) and the source, provide a link to the Creative Commons licence, and indicate if changes were made. The images or other third party material in this article are included in the article's Creative Commons licence, unless indicated otherwise in a credit line to the material. If material is not included in the article's Creative Commons licence and your intended use is not permitted by statutory regulation or exceeds the permitted use, you will need to obtain permission directly from the copyright holder. To view a copy of this licence, visit http://creativecommons.org/licenses/by/4.0/.

\section{References}

1. S. Damodaran, Food Sci. 70, 54 (2005)

2. D.J. McClements, Curr. Opin. Colloid Interface Sci. 9(5), 305-313 (2004)

3. E. Dickinson, J. Food Eng. 22(1-4), 59-74 (1994)

4. R.S.H. Lam, M.T. Nickerson, Food Chem. 141(2), 975-984 (2013)

5. J.L. Zhai, L. Day, M.I. Aguilar, T.J. Wooster, Curr. Opin. Colloid Interface Sci. 18, 257 (2013)

6. E. Dickinson, J. Dairy Sci. 80(10), 2607-2619 (1997)

7. P.N. Ezhilarasi, P. Karthik, N. Chhanwal, C. Anandharamakrishnan, Food Bioprocess Technol. 6(3), 628-647 (2013)

8. R. Murugesan, V. Orsat, Food Bioprocess Technol. 5(1), 3-14 (2012)

9. D.J. McClements, C.E. Gumus, Adv. Colloid Interf. Sci. 234, 3-26 (2016)

10. M.-T. Huang and T. Ferraro, 8 (1992)

11. K. Schwarz, E. N. Frankel, and J. B. German, 115 (1996)

12. F. Tamm, (2016)

13. H.M.C. Azeredo, K.W. Waldron, Trends Food Sci. Technol. 52, 109-122 (2016)

14. F. Song, L.M. Zhang, C. Yang, L. Yan, Int. J. Pharm. 373(1-2), 4147 (2009)
15. M. Motoki, K. Seguro, Trends Food Sci. Technol. 9(5), 204-210 (1998)

16. P. A. Rühs, C. Affolter, E. J. Windhab, and P. Fischer, J. Rheol. (N. Y. N. Y). 57, 1003 (2013)

17. S.D. Rodríguez, M. von Staszewski, A.M.R. Pilosof, Food Hydrocoll. 50, 108-115 (2015)

18. E.A. Foegeding, N. Plundrich, M. Schneider, C. Campbell, M.A. Lila, Food Hydrocoll. 72, 163-173 (2017)

19. T. Ozdal, E. Capanoglu, F. Altay, Food Res. Int. 51(2), 954-970 (2013)

20. B. Bartolomé, I. Estrella, M.T. Hernandez, Chem. Food 65, 617 (2000)

21. S. Bittner, Amino Acids 30(3), 205-224 (2006)

22. L. Jakobek, Food Chem. 175, 556-567 (2015)

23. H.M. Rawel, K. Meidtner, J. Kroll, J. Agric. Food Chem. 53(10), 4228-4235 (2005)

24. M. Ali, J. K. Keppler, T. Coenye, and K. Schwarz, J. Food Sci. 0, (2018)

25. H.M. Rawel, S. Rohn, Phytochem. Rev. 9(1), 93-109 (2010)

26. S. Rohn, Food Res. Int. 65, 13-19 (2014)

27. H.M. Rawel, D. Czajka, S. Rohn, J. Kroll, Int. J. Biol. Macromol. 30(3-4), 137-150 (2002)

28. J.E. O'Connell, P.F. Fox, Int. Dairy J. 9(8), 523-536 (1999)

29. S. Maity, S. Pal, S. Sardar, N. Sepay, H. Parvej, J. Chakraborty, U. Chandra Halder, RSC Adv. 6(113), 112175-112183 (2016)

30. F. Zhan, J. Li, Y. Wang, M. Shi, B. Li, F. Sheng, J. Agric. Food Chem. 66(26), 6832-6839 (2018)

31. M. von Staszewski, V.M. Pizones Ruiz-Henestrosa, A.M.R. Pilosof, Food Hydrocoll. 35, 505-511 (2014)

32. P. Sausse, V. Aguié-Béghin, R. Douillard, Langmuir 19(3), 737 743 (2003)

33. C.J. Beverung, C.J. Radke, H.W. Blanch, Biophys. Chem. 81(1), 59-80 (1999)

34. H. Schestkowa, T. Wollborn, A. Westphal, A. Maria Wagemans, U. Fritsching, S. Drusch, J. Colloid Interface Sci. 536, 300 (2018)

35. J. Zhai, T.J. Wooster, S.V. Hoffmann, T.H. Lee, M.A. Augustin, M.I. Aguilar, Langmuir 27(15), 9227-9236 (2011)

36. J. Zhai, A.J. Miles, L.K. Pattenden, T.H. Lee, M.A. Augustin, B.A. Wallace, M.I. Aguilar, T.J. Wooster, Biomacromolecules 11(8), 2136-2142 (2010)

37. J.K. Keppler, T. Koudelka, K. Palani, M.C. Stuhldreier, F. Temps, A. Tholey, K. Schwarz, J. Biomol. Struct. Dyn. 32(7), 1103-1117 (2014)

38. M.L. Pizarro, M. Becerra, A. Sayago, M. Beltrán, R. Beltrán, Food Anal. Methods 6(1), 123-132 (2013)

39. S. Böttcher, J.K. Keppler, S. Drusch, Colloids Surfaces A Physicochem. Eng. Asp. 518, 46-56 (2017)

40. M. Corzo-Martínez, C. Carrera Sánchez, F.J. Moreno, J.M. Rodríguez Patino, M. Villamiel, Food Hydrocoll. 27(2), 438-447 (2012)

41. P.A. Rühs, N. Scheuble, E.J. Windhab, P. Fischer, Eur. Phys. J. Spec. Top. 222(1), 47-60 (2013)

42. R. H. Ewoldt, A. E. Hosoi, and G. H. McKinley, J. Rheol. (N. Y. N. Y). 52, 1427 (2008)

43. S.E.H.J. Van Kempen, H.A. Schols, E. Van Der Linden, L.M.C. Sagis, Soft Matter 9(40), 9579 (2013)

44. J. Bergfreund, P. Bertsch, S. Kuster, P. Fischer, Langmuir 34(16), 4929-4936 (2018)

45. J. Jia, X. Gao, M. Hao, L. Tang, Food Chem. 228, 143-151 (2017)

46. Z. Wei, W. Yang, R. Fan, F. Yuan, Y. Gao, Food Hydrocoll. 45, 337-350 (2015)

47. D.C. Lange, R. Kothari, R.C. Patel, S.C. Patel, Biophys. Chem. 74(1), 45-51 (1998)

48. I. Hasni, P. Bourassa, S. Hamdani, G. Samson, R. Carpentier, H.A. Tajmir-Riahi, Food Chem. 126(2), 630-639 (2011)

49. C.D. Kanakis, I. Hasni, P. Bourassa, P.A. Tarantilis, M.G. Polissiou, H.A. Tajmir-Riahi, Food Chem. 127(3), 1046-1055 (2011)

Publisher's Note Springer Nature remains neutral with regard to jurisdictional claims in published maps and institutional affiliations. 